\title{
Protective Effect of Moutan Cortex Extract on Acetaminophen-Induced Cytotoxicity in Human Chang Liver Cells
}

\author{
Yun-Hee SHON and Kyung-Soo NAM* \\ Department of Pharmacology, College of Medicine and Intractable Disease Research Center, Dongguk University; \\ Sukjang-Dong 707, Kyongju 780-714, Korea. Received May 9, 2002; accepted July 6, 2002
}

\begin{abstract}
The aim of this study was to investigate the effect of Moutan Cortex on acetaminophen (AAP)-induced toxicity in human Chang liver cells. Cells were incubated with AAP (0-30 mM) to evaluate the drug's ability to reduce cytoviability. For the cells treated with 10, 20 and 30 mM AAP, LDH leakage was 39.8\%, 49.0\% and 57.6\%, respectively. Administration of Moutan Cortex reduced cytotoxicity in a dose-dependent manner. Glutathione (GSH) concentration in human liver cells decreased significantly after exposure to $20(p<0.05)$ and $30 \mathrm{~mm}$ $(p<0.01)$ AAP, and increased $(p<0.05)$ if incubated with AAP and Moutan Cortex. The ability of AAP to inhibit mitochondrial function and its counteraction by Moutan Cortex was also evaluated. Moutan Cortex showed dose-dependent increases in MTT metabolism and ATP levels in AAP-treated cells. The DNA content of AAPtreated cells increased with the treatment of Moutan Cortex. These observations demonstrate that Moutan Cortex may significantly attenuate AAP-induced toxicity. It can be considered a cytoprotective agent in this in vitro model of drug toxicity.
\end{abstract}

Key words acetaminophen; Moutan Cortex; glutathione; cytotoxicity; mitochondria; DNA

Acetaminophen ( $N$-acety1-p-aminophenol, paracetamol, AAP) is a widely used analgesic and antipyretic agent with very few side effects at its usual therapeutic doses. ${ }^{1)}$ However, it can cause centrilobular hepatic necrosis, renal failure, and even death in humans and experimental animals when taken in overdoses ${ }^{2}$ or in moderate doses in combination with other drugs or alcohol. ${ }^{3)}$

At therapeutic doses, AAP is primarily detoxified by glucuronidation and sulfation, ${ }^{4}$, with a small fraction metabolized by a cytochrome P450-dependent mixed function oxidase system to an electrophilic metabolite, $N$-acetyl- $p$-benzoquinoneimine (NAPQI). ${ }^{5)}$ NAPQI is normally detoxified by glutathione (GSH) and therefore displays no significant toxicity. After an overdose of AAP, the glucuronidation and sulfation routes become saturated and a larger fraction of the drug is available for metabolism by cytochrome P450, leading to rapid depletion of hepatic GSH levels. In addition to NAPQI, reactive oxygen species formed during AAP metabolism induces oxidative stress which in turn contributes to the cell injury process. AAP interferes with the ongoing processes of each and every intracellular compartment: mitochondria, ${ }^{6}$ nucleus, ${ }^{7}$ plasma membrane, ${ }^{8)}$ and cytoplasm. ${ }^{9}{ }^{9}$ Recently, another pathway of AAP toxicity was proposed the potential of AAP to influence the integrity of genomic DNA. ${ }^{10,11)}$ This may lead to cell death in the liver.

Moutan Cortex, the root cortex of Paeonia suffruticosa AndRews, is an important Chinese herbal medicine used as an analgesic, antispasmodic, and anti-inflammatory agent. The drug has long been used in remedies for female diseases. Moutan Cortex is reported to inhibit oxidative DNA cleavage $^{12)}$ and to have a scavenging effect on 1,1-diphenyl2-picrylhydrazyl (DPPH) radicals and superoxide anion radicals $\left({ }^{\circ} \mathrm{O}_{2}{ }^{-}\right)$generated by the xanthine-xanthine oxidase system. ${ }^{13)}$

In this study, we performed cytotoxicity profiles of AAP in human Chang liver cells and evaluated the protective effect of Moutan Cortex against AAP-induced toxicity by measuring the lactate dehydrogenase (LDH) leakage, GSH levels, DNA content, MTT metabolism, and ATP levels of human liver cells.

\section{MATERIALS AND METHODS}

Plant Material and Extraction Moutan Cortex was purchased from Dongguk University Oriental Medical Center in Kyongju, Korea. A voucher specimen (no. 00M-18) has been deposited in the herbarium of the Intractable Disease Research Center, Dongguk University, Kyongju, Korea. Dried Moutan Cortex (60g) was extracted with distilled water $(240 \mathrm{ml})$ for $3 \mathrm{~h}$ at room temperature. The extract was filtered, and the filtrate $(100 \mathrm{ml})$ was concentrated in vacuo and lyophilized. The lyophilized extract $(3.44 \mathrm{~g})$ of Moutan Cortex was dissolved in tissue culture medium.

Culture of Chang Liver Cells Human Chang liver cells were routinely grown in Dulbecco's modified Eagle's medium (DMEM) containing 10\% (v/v) fetal bovine serum (FBS) (complete medium), maintained at $37{ }^{\circ} \mathrm{C}$ in a humidified incubator in an atmosphere of $95 \%$ air and $5 \% \mathrm{CO}_{2}$. The medium was renewed routinely 3 and $6 \mathrm{~d}$ after passage, and when confluency was attained, cells were trypsinized and split for subcultures.

Experimental Procedure Chang cells were seeded at densities of $1.5 \times 10^{4}$ cells per well in 96-well plates in $200 \mu \mathrm{l}$ of complete medium, or $5 \times 10^{5}$ cells per well in 6-well plates in $3 \mathrm{ml}$ of complete medium, and incubated overnight for adherance. The cultures were then washed twice in warm phosphate-buffered saline (PBS) and incubated at $37^{\circ} \mathrm{C}$ in a humidified incubator in an atmosphere of $95 \%$ air and $5 \% \mathrm{CO}_{2}$ in either AAP $(0-30 \mathrm{~mm})$ or $20 \mathrm{~mm}$ AAP and Moutan Cortex extract $(0-1000 \mu \mathrm{g} / \mathrm{ml})$ for $24 \mathrm{~h}$. At the end of the incubation, cells were prepared for the various assays described below.

Lactate Dehydrogenase Leakage The release of LDH from cells over the course of the experiments was used as a measure of cytotoxicity. Briefly, cells were cultured in 96well plates and at the end of the treatment period with either AAP or AAP and Moutan Cortex extract, LDH activity in the supernatant was determined as described with the Cytotoxic- 
ity Detection Kit (LDH), (Boehringer Mannheim, Germany).

Determination of GSH Levels Chang liver cells were cultured in 96-well plates and after incubation with AAP or AAP and Moutan Cortex extract, as described above, were rinsed with PBS twice. The total GSH content in cells was assayed by an enzymatic recycling procedure ${ }^{14)}$ in which it is sequentially oxidized by 5,5'-dithiobis-(2-nitrobenzoic acid) and reduced by NADPH in the presence of glutathione. The extent of 2-nitro-5-thiobenzoic acid formation was monitored at $405 \mathrm{~nm}$. GSH content was calculated in comparison with a standard GSH curve. The protein content was determined in duplicate plates prepared and treated as described above, using a bicinchoninic protein assay kit (Sigma, St. Louis, MO, U.S.A.) with bovine serum albumin as the standard. The GSH levels were expressed as nmol/mg of protein.

DNA Assay Cells were cultured in a 6-well plate. After treatment with AAP or AAP and Moutan Cortex extract, as described above, genomic DNA was extracted by the method described by Sharma et al. ${ }^{15)}$ with minor modifications. Briefly, the cells were washed three times with PBS and harvested by incubation with $0.5 \mathrm{ml}$ proteinase $\mathrm{K}(100 \mu \mathrm{g} / \mathrm{ml})$ in $0.2 \mathrm{M}$ Tris- $0.1 \mathrm{M}$ EDTA $\left(\mathrm{pH} 8.5\right.$ ) for $10 \mathrm{~min}$ at $37^{\circ} \mathrm{C}$. The cells were transferred to $10 \%$ sodium dodecyl sulfate (SDS) solution, and incubated for $3 \mathrm{~h}$ at $55^{\circ} \mathrm{C}$. Following the addition of $75 \mu \mathrm{l}$ of $5 \mathrm{M}$ potassium acetate solution, the cells were placed on ice for $30 \mathrm{~min}$ and centrifuged for $15 \mathrm{~min}$ at $13000 \times \mathbf{g}$. To the supernatant, 2 volumes of ice-cold ethanol were added to precipitate DNA overnight at $-20^{\circ} \mathrm{C}$. The DNA was harvested by centrifugation for $20 \mathrm{~min}$ at $13000 \times \boldsymbol{g}$ in an Eppendorf microfuge, and the DNA pellet was washed with $70 \%$ ethanol and finally resuspended in $10 \mathrm{~mm}$ Tris-1 mm EDTA ( $\mathrm{pH}$ 8.0) buffer. RNA was removed by treatment with RNase A and RNase T1 at $37^{\circ} \mathrm{C}$ for $1 \mathrm{~h}$. The DNA content was determined by the absorbance at $260 \mathrm{~nm}$.

MTT Metabolism Cell mitochondrial function was quantified by the assessment of the conversion of 3-[4,5-dimethylthiazol-2-yl]-2,5-diphenyltetrazolium bromide (MTT) to a purple formazan product by mitochondrial dehydrogenase. Cells were cultured in 96-well plates. After treatment with either AAP or AAP and Moutan Cortex extract, as described above, MTT assay was performed using the method of Mosmann. ${ }^{16)}$

ATP Measurement Cells were seeded onto 6-well plates and incubated overnight at $37^{\circ} \mathrm{C}$. Fresh media containing either AAP or AAP and Moutan Cortex extract were added to the cells, then they were incubated for $0-24 \mathrm{~h}$. ATP levels were determined using an ATP Determination Kit (Promega, Madison, WI, U.S.A.) per manufacturer's instructions. Briefly, cells were harvested by scraping, pelleted at $300 \times \boldsymbol{g}$, and washed once with PBS. Cell pellets were resuspended in $100 \mu \mathrm{l}$ of boiling lysis buffer [100 mM Tris-EDTA (pH 7.7), $4 \mathrm{~mm}$ EDTA], and boiled for an additional $2 \mathrm{~min}$. Lysates were cleared at $1000 \times \boldsymbol{g}$ for $1 \mathrm{~min}$, and the supernatants were stored on ice until being measured. For ATP measurement, a $1 \mu \mathrm{l}$ sample was added to $99 \mu \mathrm{l}$ reaction buffer [luciferin, luciferase, Tris-acetate buffer ( $\mathrm{pH} 7.8)$, EDTA, magnesium acetate, bovine serum albumin, and dithiothreitol] and luminescence was measured in a luminometer (Turner Designs, Sunnyvale, CA, U.S.A.). All readings were adjusted for protein levels using a bicinchoninic protein assay kit (Sigma, St. Louis, MO, U.S.A.) with bovine serum albumin as the stan- dard. For quantitation, a standard curve was developed using $10^{-4}-10^{-12}$ M ATP.

Statistical Analysis The data were analyzed for statistical significance using Student's $t$-test. $p$ Values of less than 0.05 were considered to be significant.

\section{RESULTS}

Figure 1 shows that increasing concentrations of AAP $(0-30 \mathrm{~mm})$ caused progressively greater cytotoxicity in Chang liver cells, as demonstrated by the release of the intracellular enzyme, LDH. LDH leakage was significantly increased from $10 \mathrm{~mm}$ AAP $(39.8 \%, p<0.05)$ and was, respectively, $49.0 \%(p<0.05)$ and $57.6 \%(p<0.01)$ at 20 and $30 \mathrm{~mm}$ AAP (Fig. 1). The effect of Moutan Cortex on $20 \mathrm{~mm}$ AAPexposed Chang liver cells was evalutated by LDH leakage; the results are shown in Fig. 2. Treatment with Moutan Cortex extract prevented AAP-induced cellular damage in a dose-dependent manner (Fig. 2). Significant inhibition of AAP-induced cellular damage occurred with $1000 \mu \mathrm{g} / \mathrm{ml}$ Moutan Cortex extract.

The next series of experiments was performed to analyze AAP-induced intracellular GSH depletion in liver cells. An AAP dose-dependent depletion of intracellular GSH levels in cells was observed (Fig. 3). GSH levels were decreased to approximately $65.7 \%$ of control levels at $20 \mathrm{~mm}$ AAP and $32.5 \%$ at $30 \mathrm{~mm}$ AAP (Fig. 3). To investigate whether Moutan Cortex extract exerts its cytoprotective effect by intracellular GSH levels, we estimated GSH levels in AAP-ex-

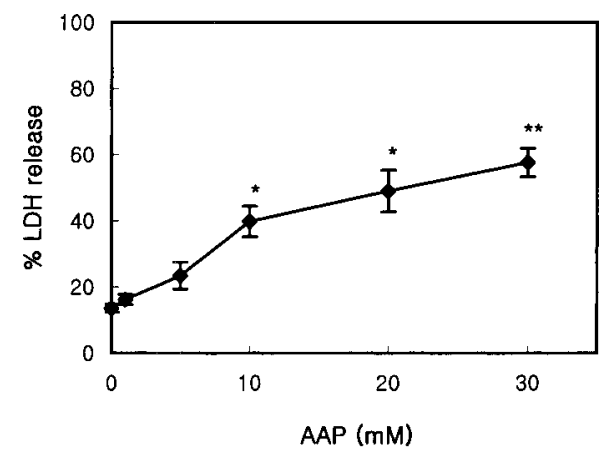

Fig. 1. Effect of Increasing Concentrations of AAP on LDH Leakage in Human Chang Liver Cells

Data shown are mean values with bars indicating the S.D. of the mean $(n=3)$. $* p<0.05, * * p<0.01$ compared with the control.

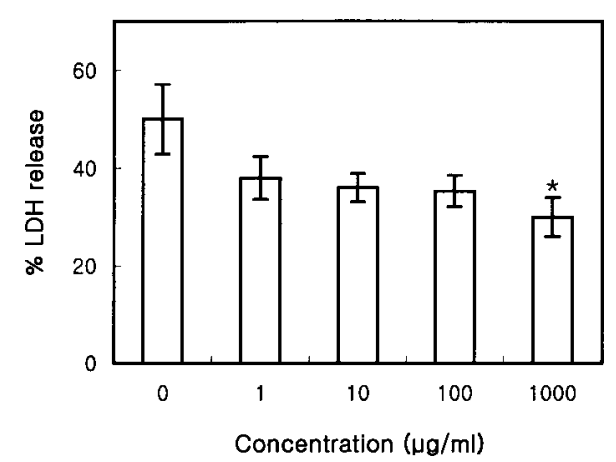

Fig. 2. Effect of Moutan Cortex Treatment on $20 \mathrm{~mm}$ AAP-Induced LDH Leakage from Human Chang Liver Cells

The values are expressed as the mean \pm S.D. of three experiments. $* p<0.05$ compared with the control. 


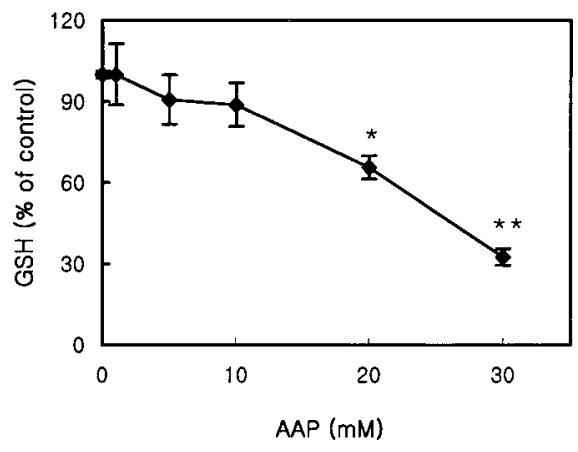

Fig. 3. Effect of Increasing Concentrations of AAP on GSH Content in Human Chang Liver Cells

Data shown are mean values with bars indicating the S.D. of the mean $(n=3)$. $* p<0.05, * * p<0.01$ compared with the control.

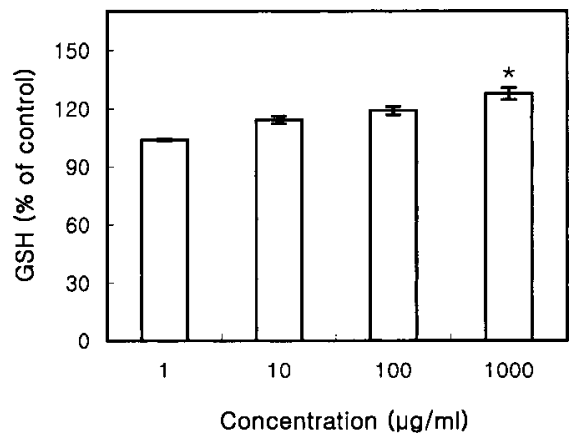

Fig. 4. Effect of Moutan Cortex Treatment on $20 \mathrm{~mm}$ AAP-Induced GSH Content from Human Chang Liver Cells

The values are expressed as the mean \pm S.D. of three experiments. $* p<0.05$ compared with the control.

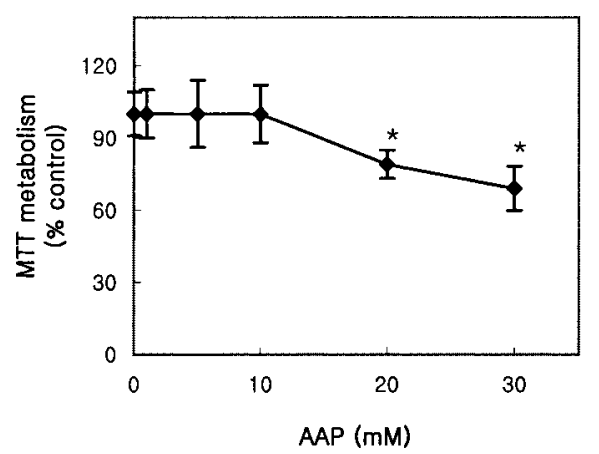

Fig. 5. Effect of Increasing Concentrations of AAP on Mitochondrial Function as Assessed by MTT Metabolism in Human Chang Liver Cells

The values are expressed as the mean \pm S.D. of three experiments. $* p<0.05$ compared with the control.

posed cells with Moutan Cortex extract treatment. In $20 \mathrm{~mm}$ AAP-treated cells with treatment of $1000 \mu \mathrm{g} / \mathrm{ml}$ Moutan Cortex extract, GSH levels was significantly different in comparison with the control (Fig. 4).

Overall mitochondrial function, as assessed by the ability to metabolize MTT, was investigated to assess cell function after incubation with AAP for $24 \mathrm{~h}$. Cells exposed to $20 \mathrm{~mm}$ AAP showed significantly decreased MTT metabolism (79.0\% of control, $p<0.05)$. Exposure to $30 \mathrm{~mm}$ AAP further decreased MTT metabolism to $69.0 \%(p<0.05)$ (Fig. 5). Cells exposed to $20 \mathrm{~mm}$ AAP and then treated with Moutan

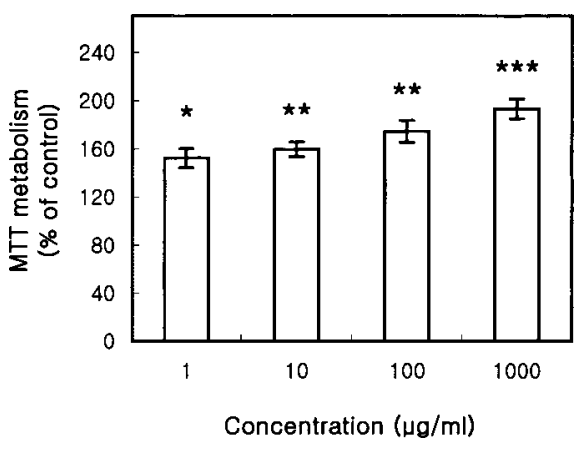

Fig. 6. Effect of Moutan Cortex Treatment on $20 \mathrm{~mm}$ AAP-Induced Mitochondrial Function as Assessed by MTT Metabolism from Human Chang Liver Cells

Data shown are mean values with bars indicating the S.D. of the mean $(n=3)$. $* p<0.05, * * p<0.01, * * * p<0.005$ compared with the control.

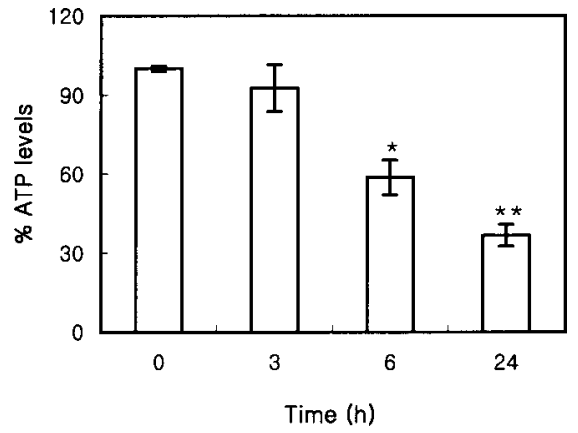

Fig. 7. Effect of AAP $(20 \mathrm{~mm})$ on Intracellular ATP Levels in Human Chang Liver Cells

The values are expressed as the mean \pm S.D. of three experiments. $* p<0.05$, ** $p<0.01$ compared with the control.

Cortex extract $(1-1000 \mu \mathrm{g} / \mathrm{ml})$ showed a significant increase in MTT metabolism (Fig. 6).

It has been suggested ${ }^{17,18)}$ that the most important early event in AAP toxicity is the inhibition of mitochondrial function, which consequently leads to a depletion of cellular ATP. We investigated whether the cell damage produced by AAP was associated with alterations in intracellular ATP levels. Our results indicate that lower concentrations of AAP $(1,5$, $10 \mathrm{~mm}$ ) had little effect on ATP levels in Chang liver cells at any of the time points examined (data not shown). We observed a decrease in ATP levels induced by $20 \mathrm{~mm}$ AAP that began within $6 \mathrm{~h}(58.6 \%)$, and was reduced to $36.6 \%$ of the control by $24 \mathrm{~h}$ (Fig. 7). Moutan Cortex extract showed a dose-dependent increase in ATP levels in $20 \mathrm{~mm}$ AAP-treated cells (Fig. 8).

Exposure to AAP also caused a dose-dependent decrease in the DNA content of liver cells (data not shown). DNA content in cells treated with $20 \mathrm{~mm}$ AAP plus $100(p<0.05)$ and $1000 \mu \mathrm{g} / \mathrm{ml}(p<0.01)$ Moutan Cortex extract was significantly increased (Fig. 9).

\section{DISCUSSION}

We have investigated the in vitro cytoprotective role of Moutan Cortex in cultured human liver cells against AAP-induced toxicity. The results of the present study demonstrate that treatment of liver cells with Moutan Cortex extract had a markedly protective effect against AAP-induced toxicity. A 


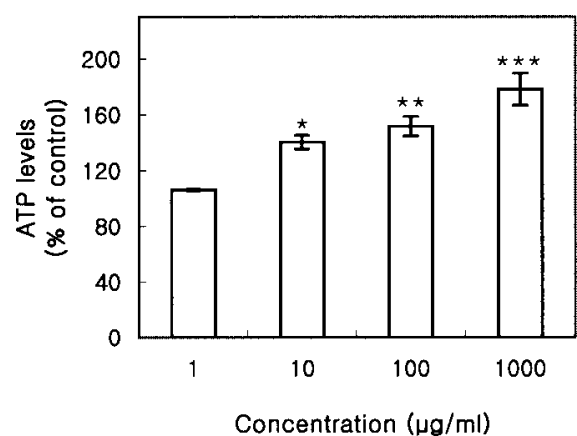

Fig. 8. Effect of Moutan Cortex Treatment on $20 \mathrm{~mm}$ AAP-Induced Intracellular ATP Levels from Human Chang Liver Cells

Data shown are mean values with bars indicating the S.D. of the mean $(n=3)$. $* p<0.05, * * p<0.01, * * * p<0.005$ compared with the control.

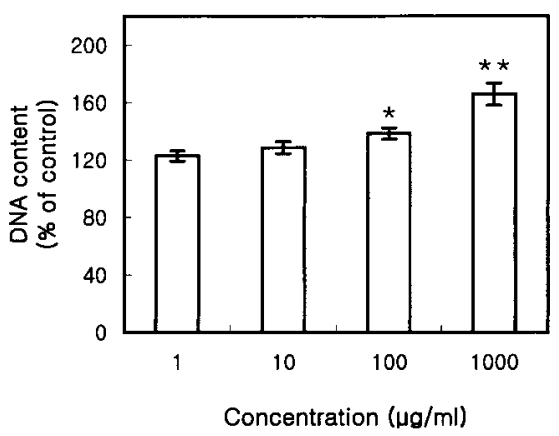

Fig. 9. Effect of Moutan Cortex Treatment on $20 \mathrm{~mm}$ AAP-Induced DNA Content from Human Chang Liver Cells

The values are expressed as the mean \pm S.D. of three experiments. $* p<0.05$, $* * p<0.01$ compared with the control.

study on the mechanisms of AAP-induced toxicity showed that GSH plays a key role in the detoxification of the reactive toxic metabolites of AAP, and that liver necrosis begins when GSH stores are markedly depleted. ${ }^{19)}$ Similarly, depletion of $\mathrm{GSH}$ is a prerequisite for the expression of AAP-induced damage in murine hepatocytes. ${ }^{17)}$ The toxicity of AAP to cells in our study may be attributed to its metabolism into a reactive metabolite (NAPQI). The following hypothesis could be proposed. The depletion of GSH observed in our model renders the cell vulnerable to attack by NAPQI. Since NAPQI is an electrophilic oxidant species, it may induce arylation and oxidation of protein and thiol groups within the cell. In turn, this phenomenon may cause a loss of protein integrity and associated enzyme function, leading to cell death. $^{5,20)}$ Our results showed that treatment with Moutan Cortex $(1000 \mu \mathrm{g} / \mathrm{ml})$ significantly reduced AAP-induced GSH depletion (Fig. 4). This result is probably due to the inhibition of the bioactivation of AAP by Moutan Cortex resulting in the decreased formation of NAPQI.

However, AAP-depleted GSH levels partially recovered with exposure to Moutan Cortex. Thus, it is less likely that Moutan Cortex acts directly on GSH, as does $\mathrm{N}$-acetylcysteine, which is reported to prevent a fall in GSH levels in AAP-treated hepatocytes by acting as a cysteine precursor. $^{21,22)}$ The limited restoration of GSH levels effected by Moutan Cortex suggests that GSH depletion may not be the sole mechanism in cell protection, but rather one factor in the general cytoprotective influence of this compound noted in this in vitro study.

Our data suggest that the cytotoxicity induced by AAP to Chang liver cells depends on dose. The damage is targeted to a specific cellular compartment, the mitochondria, as shown by the MTT assay (Fig. 5) and ATP measurement (Fig. 7). Mitochondria are recognized as a potential target of drug-induced toxicity. It has been suggested ${ }^{17)}$ that the most important early event in AAP toxicity is the inhibition of mitochondrial function, which consequently leads to a depletion of cellular ATP. Several investigators ${ }^{23-25)}$ reported recently that intracellular ATP levels play an important role in cell death that will result from a pathological insult. It has been demonstrated that depleting ATP levels to less than $50 \%$ of control cultures is sufficient for the inhibition of apoptosis and leads to necrotic cell death. ${ }^{26)}$ Alternatively, hepatocellular necrotic cell death has been observed following only a $10-15 \%$ reduction in ATP levels. ${ }^{27,28)}$ Thus, another possible explanation for the cytoprotection by Moutan Cortex against AAP-induced toxicity is that mitochondrial damage is diminished, as shown by the ability of cells to metabolize MTT and reverse the depletion of intracellular ATP levels.

Stability of the cellular genomic apparatus is constantly challenged by a wide-spectrum of exogenous (environmental toxicants, high energy radiation) and endogenous (oxidative damage, factor imbalance) threats, which generate DNA lesions. The experiments with AAP have shown that this hepatotoxic analgesic directly alkylates genomic DNA both in vivo and in vitro, and produces genotoxic effects in varied test systems. ${ }^{29)}$ These findings have been extended to show that AAP exposure causes the inhibition of DNA synthesis and increases single-strand breaks and sister chromatid exchanges in V79 Chinese hamster cells. ${ }^{30)}$ Since AAP is known to inhibit systems that maintain DNA integrity, it is possible that the capacity of AAP to damage DNA is modulated by Moutan Cortex.

In summary, the protective potential of Moutan Cortex against drug-induced toxicity in human Chang liver cells could be attributed to the following mechanisms: (a) inhibition of the decrease in GSH, with a resultant increase in the availability of this agent for cell detoxification, (b) prevention of mitochondrial damage, or (c) protection of DNA integrity. These mechanisms may be associated with membrane stabilization and restoration of intracellular GSH levels, and could be responsible for the cytoprotective potential in our in vitro model. However, further studies are necessary to elucidate the mechanism and to compare the AAP-induced toxicity in an animal model, as well as the inhibition of the toxicity associated with Moutan Cortex.

Acknowledgment This work is supported by the Dongguk University research fund.

\section{REFERENCES}

1) Mitchell J. R., N. Engl. J. Med., 319, 1601-1602 (1988).

2) Boyd E. M., Bereczky G. M., Br. J. Pharmacol., 26, 606-614 (1996).

3) Seeff L. B., Cuccherini B. A., Zimmerman H. J., Adler E., Benjamin S. B., Ann. Int. Med., 104, 399-404 (1986).

4) Nelson S. D., J. Med. Chem., 25, 753 -765 (1982).

5) Albano E., Rundgren M., Harvison P. J., Nelson S. D., Moldeus P., Mol. Pharmacol., 28, 306-311 (1985).

6) Gupta S., Rogers L. K., Taylor S. K., Smith C., Toxicol. Appl. Pharma- 
col., 146, 317-327 (1997).

7) Ray S. D., Mumaw V., Raie R. R., Fariss M. W., J. Pharmacol. Exp. Ther., 279, 1470-1483 (1996).

8) Moore M., Thor H., Moore G., Nelson S., Moldeus P., Orrenius S., J. Biol. Chem., 260, 13035-13040 (1985).

9) Pumford N. R., Halmes N. C., Martin B. M., Cook R. J., Wagner C., Hinson J. A., J. Pharmacol. Exp. Ther, 280, 501-505 (1997).

10) Ray S. D., Sorge C. L., Tavacoli A., Raucy J. L., Corcoran G. B., Adv. Exp. Med. Biol., 238, 699-705 (1991).

11) Ray S. D., Kamendulis L. M., Gurule M. W., Yorkin R. D., Corcoran G. B., FASEB J., 7, 453-463 (1993).

12) Okubo T., Nagai F., Seto T., Satoh K., Ushiyama K., Kano I., Biol. Pharm. Bull., 23, 199-203 (2000).

13) Yoshikawa M., Ohta T., Kawaguchi A., Matsuda H., Chem. Pharm. Bull., 48, 1327-1331 (2000).

14) Meister A., Anderson M., Ann. Rev. Biochem., 52, 711-760 (1983).

15) Sharma S., Stutzman J. D., Kelloff G. J., Steele V. E., Cancer Res., 54, 5848-5855 (1994).

16) Mosmann T., J. Immunol. Methods, 65, 55-63 (1983).

17) Burcham P. C., Harman A. W., J. Biol. Chem., 266, 5049-5054 (1991).

18) Meyers L. L., Beierschmitt W. P., Khairallah E. A., Cohen S. D., Toxicol. Appl. Pharmacol., 93, 378-387 (1988).
19) Mitchell J. R., Jollow D. J., Potter W. Z., Davis D. C., Gillette J. R., Brodie B. B., J. Pharmacol. Exp. Ther, 187, 211-217 (1973).

20) Porubek D. J., Rundgren M., Harvison P. J., Nelson S. D., Moldeus P., Mol. Pharmacol., 31, 647-653 (1987).

21) Harman A. W., Self G., Toxicology, 41, 83-93 (1986).

22) Massey T. E., Racz W. J., Toxicol. Appl. Pharmacol., 60, 220-228 (1981).

23) Ha H. C., Snyder S. H., Proc. Natl. Acad. Sci. U.S.A., 96, 1397813982 (1999).

24) Nicotera P., Leist M., Fernanado-May E., Toxicol. Lett., 102, 139-142 (1998).

25) Qian T., Herman B., Lemasters J. J., Toxicol. Appl. Pharmacol., 154, $117-125$ (1999).

26) Leist M., Single B., Castoldi A. F., Kuhnle S., Nicotera P., J. Exp. Med., 185, 1481-1486 (1997).

27) Nieminen A. L., Saylor A. K., Testfai S. A., Herman B., Lemasters J. J., Am. J. Physiol., 267, C67-C74 (1994).

28) Qian T., Nieminen A. L., Herman B., Lemasters J. J., Am. J. Physiol., 273, C1783-C1792 (1997).

29) Dybing E., Holme J. A., Gordon W. P., Soderlund E. J., Dahlin D., Nelson S. D., Mutat. Res., 108, 21-32 (1984).

30) Hongslo J. K., Ghristensen T., Brunborg G., Biornstad C., Holme J. A., Mutat. Res., 204, 333-341 (1998). 